\title{
Implementação Prática de um PID Robusto Sintonizado via LMIs: Controle de Velocidade de um Motor C.C.
}

\author{
Ruhan P. Policarpo de Souza* Emerson R. Pires da Silva* \\ Luís Francisco S. Buzachero* João Marcos M. Bonzanini * \\ * Laboratório de Controle e Otimização de Sistemas, Universidade \\ Tecnológica Federal do Paraná - Câmpus Cornélio Procópio, PR \\ (e-mails: ruhan.policarpo@hotmail.com,emersonr@utfpr.edu.br, \\ luizf@utfpr.edu.br,joao_mmb20@yahoo.com.br).
}

\begin{abstract}
The DC motor (Direct Current), widely used in modern industry, can suffer parametric variations during it's lifetime. Therefore, a previously designed control system with no-robustness characteristics can lose efficiency over time and even make the system unstable. Considering a PID controller (Proportional, Integral and Derivative), most used control structure in the current industry, this article presents the practical implementation of a robust PID controller, applied in a DC motor speed control. The controller's gain, $K_{p}, K_{i}$ and $K_{d}$, are tuned through LMIs (Linear Matrix Inequalities), a technique that easily allows uncertain parameters treatment. The state feedback LMIs were used through the decay rate, guaranteeing system performance over robust pole allocation. The implemented control system proved to be viable under different conditions, even under feedback failure.

Resumo: O motor CC (Corrente Contínua), amplamente utilizado na indústria moderna, pode sofrer variações paramétricas durante sua vida útil. Logo, um sistema de controle previamente projetado sem características de robustez pode perder eficiência no decorrer de um período de tempo e, até mesmo, tornar o sistema instável. Considerando um controlador PID (Proporcional, Integral e Derivativo), estrutura de controle mais utilizada na indústria atual, este trabalho apresenta a implementação prática de um controlador PID robusto, aplicado no controle de velocidade de um motor CC. Os ganhos do controlador, $K_{p}, K_{i}$ e $K_{d}$ são sintonizados por meio de LMIs (Linear Matrix Inequalities), técnica que permite facilmente o tratamento de parâmetros incertos no modelo. Utilizou-se para a sintonia do PID robusto, LMIs de realimentação de estados via taxa de decaimento, garantindo desempenho ao sistema por meio da alocação robusta de polos. O sistema de controle implementado se mostrou viável em condições diversas, diante até mesmo de operação com falha na realimentação.
\end{abstract}

Keywords: PID Robust Control; LMI, DC Motor, Decay-Rate.

Palavras-chaves: Controle PID Robusto; LMI; Motor CC; Taxa de Decaimento.

\section{INTRODUÇÃO}

Nos métodos de manufatura e processos indústriais atuais, uma técnica de controle amplamente utilizada é o controle PID (Proporcional, Integral e Derivativo). Sua vasta utilização industrial se deve a grande faixa de aplicação e sua facilidade de implementação (Ogata, 2010). Ainda, sabe-se que o controle PID é responsável por mais de 97\% do controle industrial nas áreas químicas, refinarias e papel e celulose, mesmo sabendo que apenas cerca de $36 \%$ apresentam desempenho excelente ou aceitável (Cheng, 2006). Dentre os diversos processos e equipamentos ao qual o controle PID pode ser aplicado, destaca-se o motor de corrente contínua (CC). Possuindo fácil operação e controle, é utilizado em aplicações que exigem controle de velocidade, posição e torque, como, por exemplo, máquinas operatrizes, prensas, máquinas da indústria têxtil, moagem e esteiras em geral.
Os motores, assim como diversos equipamentos, estão sujeitos a desgastes dentro do ambiente industrial, causados por fenômenos como vibrações, elevações de temperatura e presença de atmosfera contaminante. Logo, com o desgaste, os parâmetros da máquina podem sofrer alterações e, consequentemente, um sistema de controle anteriormente aplicado pode não atender as especificações iniciais de projeto, representando um grande problema da engenharia. A alteração nos parâmetros pode prejudicar o desempenho dinâmico de um sistema de controle, e, até mesmo, torná-lo instável. Para evitar essas complicações, projetos robustos podem ser utilizados na abordagem do problema. Diversos trabalhos podem ser encontrados na literatura especializada, como em Zhangbao et al. (2015), que apresenta um método de controle robusto via observador de distúrbios, aplicado a um motor CC. Bensafia et al. (2015) por sua vez, apresenta uma técnica de controle robusto utilizando um controlador PI adaptativo, entre outros. 
Dado um sistema de controle robusto, a sintonia de seus parâmetros e a adequação do desempenho dinâmico do sistema aos requisitos de projeto constituem uma tarefa complexa, contudo, a utilização de desigualdades matriciais lineares (LMIs, acrônimo inglês para Linear Matrix Inequalities) simplifica o tratamento de incertezas no modelo, e facilita a inclusão de índices de desempenho no âmbito do projeto (Boyd et al., 1994). Com a expansão dos métodos computacionais e a criação de ferramentas específicas, sua utilização se tornou mais simples e acessível. Em trabalhos recentes utilizando LMIs, Hypiusová and Rosinová (2018) apresenta uma metodologia para alocação robusta de polos utilizando LMI, aplicado a um levitador magnético, tal como Chairinnas et al. (2016), que utiliza a abordagem das LMIs para o desenvolvimento de controladores robustos para modelos com incertezas paramétricas.

Neste trabalho propõe-se a obtenção de resultados práticos por meio da implementação de um controlador PID robusto aplicado em um motor CC sujeito a incertezas no modelo. A técnica utilizada via LMIs para a sintonia dos ganhos do PID robusto possibilita que o projetista aloque os polos de malha-fechada em uma região $\gamma$-estável. Além de garantir estabilidade, propicia bom desempenho dinâmico ao sistema controlado.

\section{REALIMENTAÇÃO DE ESTADOS EM SISTEMAS LINEARES INCERTOS}

Considere um sistema representado por espaços de estados, dado pela Equação (1), contendo incertezas invariantes no tempo:

$$
\dot{x}(t)=A(\alpha) x(t)+B(\alpha) u(t) .
$$

Neste caso, $A(\alpha) \in \mathbb{R}^{n \times n}$ e $B(\alpha) \in \mathbb{R}^{n \times m}$ são as matrizes contendo os coeficientes do sistema, $x(t) \in \mathbb{R}^{n}$ o vetor de estados e $u(t) \in \mathbb{R}^{m}$ o sinal de controle. Entretanto, os coeficientes $(A, B)(\alpha)$ do sistema não são precisamente conhecidos, pertencendo a um politopo dado por:

$$
(A, B)(\alpha)=\sum_{j=1}^{r} a_{j}(A, B)_{j},
$$

sendo $r=2^{\phi}$, com $\phi$ o número de incertezas nas matrizes $A$ e $B$, e $(A, B)_{j}$ representando os vértices do respectivo politopo. As constantes reais desconhecidas, $\alpha_{j}$, pertencem a um simplex $\mathscr{S}$ definido por:

$$
\mathscr{S}=\left\{\sum_{j=1}^{r} \alpha_{j}=1, \quad \alpha_{j} \geq 0, \quad j=1,2, \ldots, r\right\} .
$$

Portanto, representando o sistema pela combinação convexa de seus vértices, temos:

$$
\dot{x}(t)=\sum_{j=1}^{r} a_{j}\left(A_{j} x(t)+B_{j} u(t)\right) .
$$

No sistema, aplica-se um sinal de controle, realimentando a entrada do sistema à partir de seus estados, à ser dado respectivamente por:

$$
u(t)=-K x(t)
$$

A matriz $K \in \mathbb{R}^{m \times n}$, contém os ganhos do controlador. Busca-se então uma matriz $K$ tal qual o sistema em malha fechada torne-se estável. Substituindo a Equação (5) na Equação (4), obtêm-se a equação do sistema realimentado:

$$
\dot{x}(t)=\sum_{j=1}^{r} a_{j}\left(A_{j}-B_{j} K\right) x(t) .
$$

Sendo assim, o ajuste dos parâmetros da matriz $K$ tem a função de adequar o comportamento do sistema frente ao desempenho e características desejadas pelo projetista.

\section{CONDIÇÕES PARA A $\gamma$-ESTABILIDADE ROBUSTA}

A representação do sistema em desigualdades matriciais lineares permite com facilidade a inserção de índices de desempenho, objetivando, na maioria das vezes, restrigir a localização dos polos de malha fechada à uma região desejada, alterando o comportamento dinâmico do sistema. Uma técnica conhecida é a inclusão da taxa de decaimento (Boyd et al., 1994). A taxa de decaimento ( $\gamma$-estabilidade), também chamada de maior expoente de Lyapunov, é definida como sendo a maior constante positiva $\gamma$, que se mantenha para todo $x(t), t>0$, tal que :

$$
\lim _{t \rightarrow \infty} e^{\gamma t}\|x(t)\|=0 .
$$

A restrição na taxa de decaimento pode ser usada para diminuir o tempo de estabelecimento do sistema, já que os polos de malha fechada são alocados em uma região mais deslocada em relação ao eixo imaginário. A alocação dos polos é dada na região cinza, conforme Figura 1.

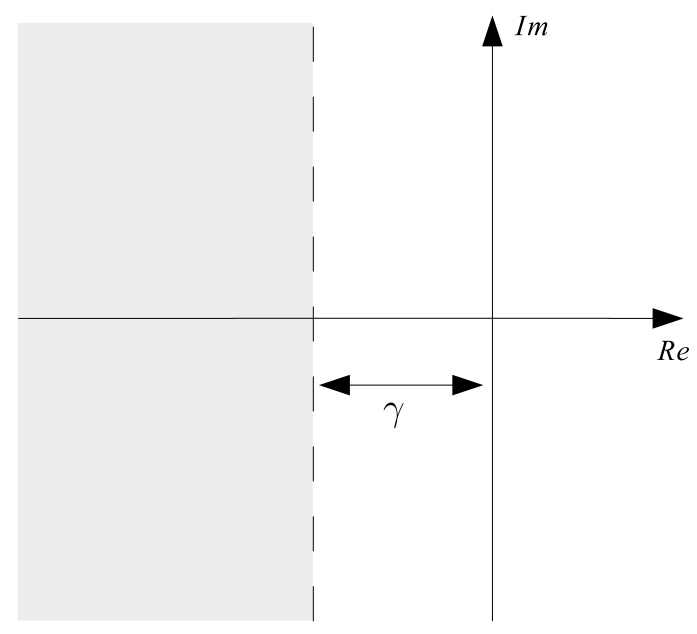

Figura 1. Região de alocação dos polos via $\gamma$-estabilidade.

A partir dessas informações, o Lema 1 apresenta condições robustas para que o sistema incerto (6) seja $\gamma$-estável.

Lema 1. O sistema (6) é $\gamma$-estável se existir uma matriz simétrica $P \in \mathbb{R}^{n \times n}$ e uma matriz $G \in \mathbb{R}^{m \times n}$, tal que as LMIs abaixo sejam satisfeitas, dada uma taxa de decaimento $\gamma>0$.

$$
A_{j} P-B_{j} G+P A_{j}^{\prime}-G^{\prime} B_{j}^{\prime}+2 \gamma P<0,
$$




$$
P>0
$$

sendo $K=G P^{-1}$ o ganho de realimentação de estados robusto.

Prova. Veja Boyd et al. (1994).

\section{SÍNTESE DO PID ROBUSTO VIA REALIMENTAÇÃO DE ESTADOS}

Considere um sistema genérico de segunda ordem com incertezas paramétricas para o desenvolvimento do controlador PID robusto via espaço de estados (Ge et al., 2002):

$$
G(s)=\frac{b_{0}}{s^{2}+a_{1} s+a_{0}},
$$

sendo os parâmetros incertos representados pelas variáveis contidas dentro dos intervalos:

$$
a_{1} \in\left[\underline{a_{1}}, \overline{a_{1}}\right], a_{0} \in\left[\underline{a_{0}}, \overline{a_{0}}\right], b_{0} \in\left[\underline{b_{0}}, \overline{b_{0}}\right] .
$$

Neste conjunto, $a_{n}$ e $\overline{a_{n}}$ representam os limitantes mínimos e máximos de cada variável. O sinal de controle de um PID pode ser definido como:

sendo:

$$
u(t)=K_{p} e(t)+K_{d} \frac{d e(t)}{d t}+K_{i} \int_{0}^{t} e(t) d t
$$

$$
e(t)=r(t)-y(t) .
$$

Considera-se as seguintes equivalências de estados:

$$
\begin{gathered}
x_{1}(t)=y(t) \\
x_{2}(t)=\dot{x_{1}}(t) \\
x_{3}(t)=-\int_{0}^{t}(r(t)-y(t)) d t .
\end{gathered}
$$

Substituindo (11) em (10) e realizando as transformações (12), (13) e (14), chega-se em:

$$
u(t)=K_{p} r(t)-K_{p} x_{1}(t)+K_{d} \frac{d r(t)}{d t}-K_{d} x_{2}(t)-K_{i} x_{3}(t) .
$$

Reorganizando os termos, obtêm-se:

$$
u(t)=-K x(t)+K_{p} r(t)+K_{d} \dot{r}(t),
$$

sendo $K=\left[K_{p}, K_{d}, K_{i}\right]$ e $x(t)=\left[x_{1}(t), x_{2}(t), x_{3}(t)\right]^{T}$. O sistema de controle completo pode ser representado na forma matricial aumentada, pelas seguintes equações:

$\left[\begin{array}{l}\dot{x_{1}}(t) \\ \dot{x_{2}}(t) \\ \dot{x_{3}}(t)\end{array}\right]=\left[\begin{array}{ccc}0 & 1 & 0 \\ -a_{0} & -a_{1} & 0 \\ 1 & 0 & 0\end{array}\right]\left[\begin{array}{l}x_{1}(t) \\ x_{2}(t) \\ x_{3}(t)\end{array}\right]+\left[\begin{array}{c}0 \\ b_{0} \\ 0\end{array}\right] u(t)+\left[\begin{array}{c}0 \\ 0 \\ -1\end{array}\right] r(t)$,

$$
u(t)=-\left[\begin{array}{lll}
K_{p} & K_{d} & K_{i}
\end{array}\right]\left[\begin{array}{l}
x_{1}(t) \\
x_{2}(t) \\
x_{3}(t)
\end{array}\right]+K_{p} r(t)+K_{d} \dot{r}(t),
$$

$$
y(t)=\left[\begin{array}{lll}
1 & 0 & 0
\end{array}\right]\left[\begin{array}{l}
x_{1}(t) \\
x_{2}(t) \\
x_{3}(t)
\end{array}\right] .
$$

Nesta representação, o controlador PID robusto pode ser sintonizado por realimentação estática dos estados. Através do Lema (1), é possível encontrar um ganho robusto $K$ que satisfaz todo o conjunto de incertezas. A Figura 2 representa o sistema de controle equivalente.

Os 3 parâmetros incertos, dados em (9), formarão as matrizes incertas $A_{j}$ e $B_{j}$ do sistema. O conjunto politópico $\left(2^{3}=8\right.$, sendo 3 o número de incertezas) será dado por:

$$
\Omega=\operatorname{Cov}\left\{\left[A_{1}, B_{1}\right],\left[A_{2}, B_{2}\right], \ldots,\left[A_{8}, B_{8}\right]\right\} .
$$

\section{IMPLEMENTAÇÃO PRÁTICA DO PID ROBUSTO}

O PID robusto será aplicado no controle de velocidade de um motor CC, e foi organizado com a seguinte estrutura:

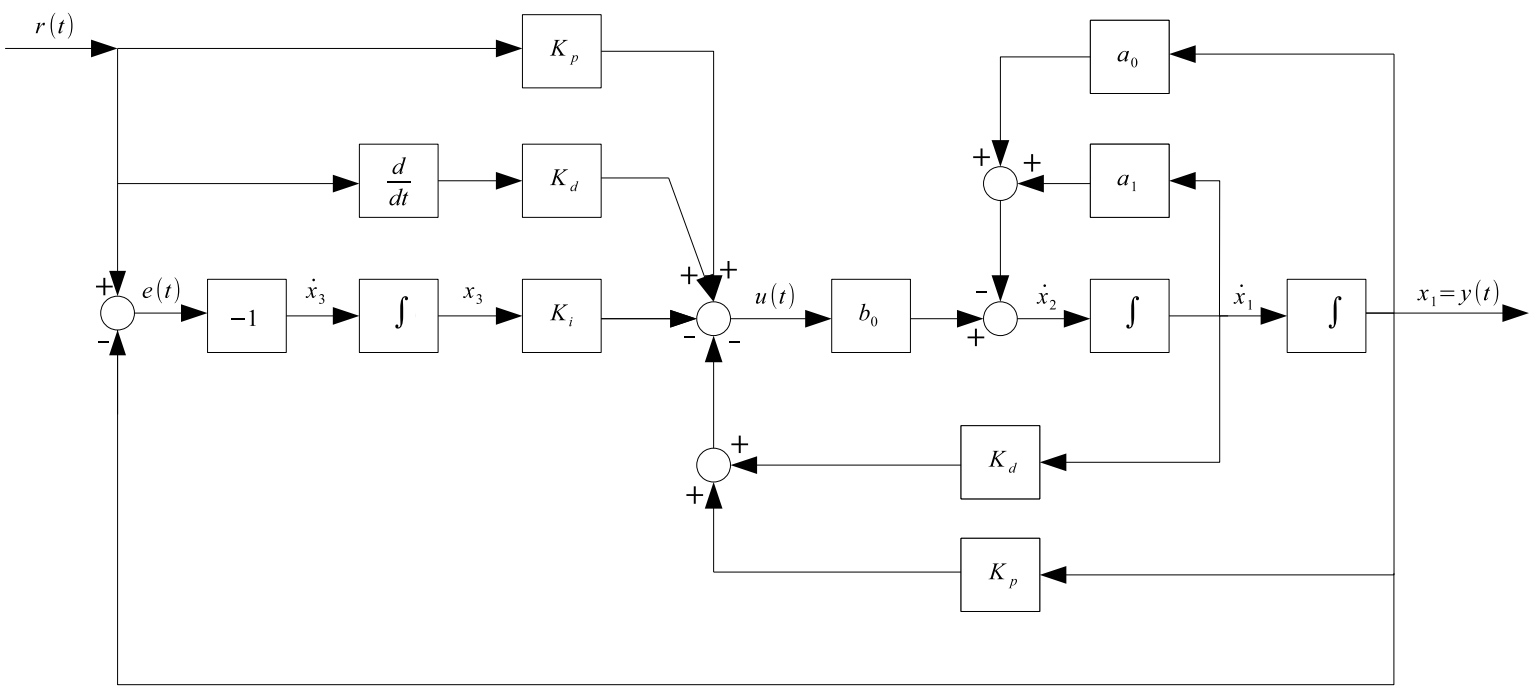

Figura 2. Sistema de controle PID robusto. 
identificação dos parâmetros do motor, modelagem do PID robusto e projeto do controlador $K$.

Para a implementação, buscou-se utilizar um módulo didático de baixo custo, simples e de fácil acesso, a fim de permitir a reprodutibilidade do sistema. O módulo foi previamente desenvolvido por Bonzanini (2017), e pode ser visto na Figura 3.

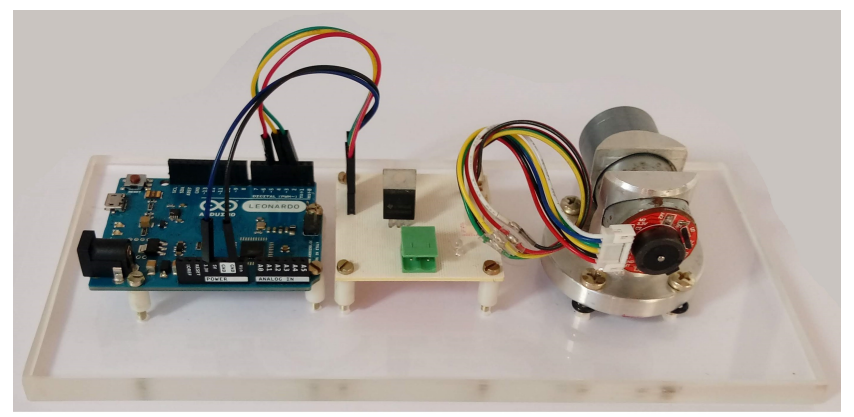

Figura 3. Módulo didático utilizado para a implemetação do PID robusto no controle do motor CC.

No módulo desenvolvido, o motor CC é alimentado por um driver de alimentação, acionado pela placa comunicadora Arduino ${ }^{\circledR}$. Por sua vez, o controle e o processamento de dados é realizado pelo software Simulink ${ }^{\circledR}$, com base no sinal de velocidade lido pelo encoder do motor. O fluxograma do módulo pode ser visto na Figura 4.

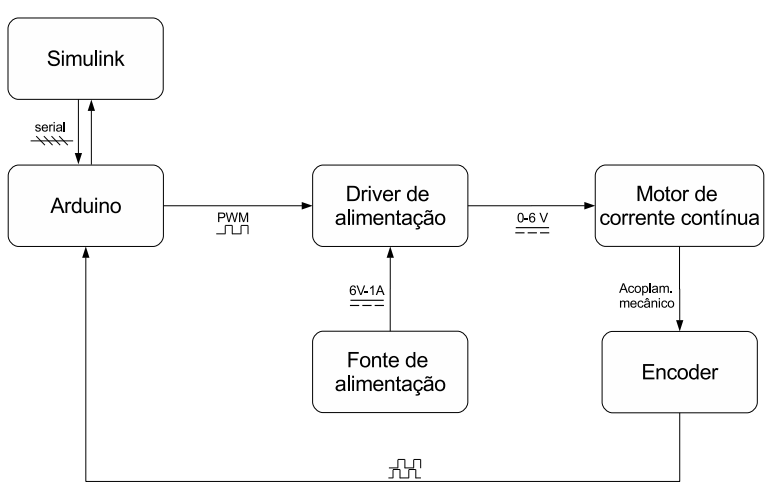

Figura 4. Fluxograma de implementação

\subsection{Identificação dos parâmetros do motor}

Baseia-se em observar a curva de reação de velocidade do motor, diante da aplicação de um sinal do tipo degrau e, então, estimar seus parâmetros. Dada a sensibilidade do sistema e a alta taxa de ruído, optou-se pela aplicação do método em malha fechada. Realizou-se medições considerando toda faixa de atuação do motor, com intervalos de $50 \mathrm{rpm}$. Iniciou-se em $100 \mathrm{rpm}$, devido a dificuldade na partida pelo baixo nível de tensão, e executou-se até 350 rpm, garantindo a validade dos parâmetros em casos de overshoot e ampliando a área de parametrização do motor. Observou-se o comportamento transitório, em especial o tempo de pico, $T_{p}$, máximo sobressinal, $M_{o}$, e o valor de saída em regime permanente. Os resultados estão na Tabela 1, e a curva de reação $n^{\circ} 3$ pode ser vista na Figura 5 .
Tabela 1. Comportamento transitório de malha fechada.

\begin{tabular}{ccccc}
\hline & $\begin{array}{c}\mathrm{R}(\mathrm{s}) \\
\mathrm{n}\end{array}$ & $\begin{array}{c}T_{p} \\
{[\mathrm{rpm}]}\end{array}$ & $\begin{array}{c}M_{o} \\
{[\mathrm{~s}]}\end{array}$ & $\begin{array}{c}\lim _{t \rightarrow \infty} y(t) \\
{[\mathrm{rpm}]}\end{array}$ \\
\hline 1 & 100 & 0,145 & 42,18 & 63,06 \\
2 & 150 & 0,155 & 42,22 & 105,63 \\
3 & 200 & 0,130 & 45,31 & 143,24 \\
4 & 250 & 0,140 & 36,85 & 183,36 \\
5 & 300 & 0,145 & 25,94 & 221,98 \\
6 & 350 & 0,140 & 20,99 & 255,69 \\
\hline
\end{tabular}

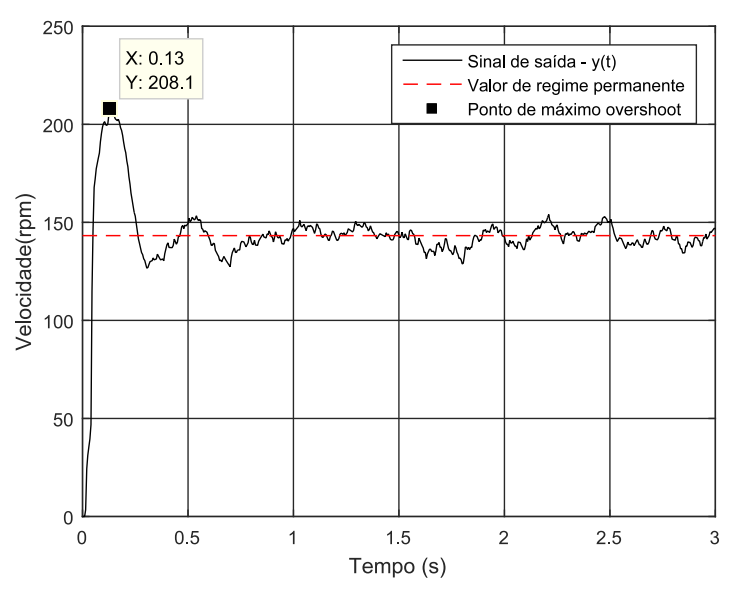

Figura 5. Curva de reação $\mathrm{n}^{\circ} 3$

Com base nos valores apresentados na Tabela 1 , foi possível estimar a frequência natural não amortecida, $\omega_{n}$, e o coeficiente de amortecimento $\zeta$ do sistema de malha fechada. A partir de uma simples manipulação matemática, os coeficientes de malha-aberta, $G(s)$, são obtidos e apresentados na Tabela 2 .

Tabela 2. Coeficientes de malha aberta $G(s)$.

\begin{tabular}{cccc}
\hline $\mathrm{n}$ & $b_{0}$ & $a_{1}$ & $a_{0}$ \\
\hline 1 & 19100 & 11,91 & 186,5 \\
2 & 18670 & 11,13 & 130,6 \\
3 & 26690 & 12,18 & 176,2 \\
4 & 24400 & 14,26 & 147,8 \\
5 & 24690 & 18,61 & 144,6 \\
6 & 27520 & 22,30 & 169,2 \\
\hline
\end{tabular}

Considerando a variação dos parâmetros, verifica-se que um parâmetro não é precisamente conhecido, mas, pertencente a um politopo de incertezas. De (9), pode-se considerar portanto que:

$$
\begin{aligned}
& 18670 \leqslant b_{0} \leqslant 27520, \\
& 11,13 \leqslant a_{1} \leqslant 22,30, \\
& 130,6 \leqslant a_{0} \leqslant 186,5 .
\end{aligned}
$$

5.2 Obtenção dos vértices do politopo com inclusão de falha no sinal de realimentação

Aplicou-se a modelagem apresentada na Seção 4, além de adicionar o conceito de falha na realimentação. Podese considerar uma falha no atuador do sistema inserindo na matriz $B$ uma constante $F_{r}$, a atenuar o sinal de realimentação. Com isso, a incerteza do parâmetro $b_{0}$ 
terá um novo valor mínimo, dada a possível atenuação de realimentação. O projeto do controlador levará em consideração uma falha de até $30 \%$ na realimentação do sistema, logo:

$$
0,7 \leq F_{r} \leq 1
$$

O sistema (17) torna-se:

$$
\left[\begin{array}{l}
\dot{x_{1}}(t) \\
\dot{x_{2}}(t) \\
\dot{x_{3}}(t)
\end{array}\right]=\left[\begin{array}{ccc}
0 & 1 & 0 \\
-a_{0} & -a_{1} & 0 \\
1 & 0 & 0
\end{array}\right]\left[\begin{array}{l}
x_{1}(t) \\
x_{2}(t) \\
x_{3}(t)
\end{array}\right]+\left[\begin{array}{c}
0 \\
F_{r} b_{0} \\
0
\end{array}\right] u(t)+\left[\begin{array}{c}
0 \\
0 \\
-1
\end{array}\right] r(t)
$$

As matrizes incertas $A_{j}$ e $B_{j}$ são definidas a partir das combinações resultantes entre os vértices de cada parâmetro, garantindo a robustez em todos os possíveis pontos do motor. A quantidade de modelos é dado por $2^{\phi}$, sendo $\phi$ o número de parâmetros incertos. Neste projeto, $\phi=3$, logo, tem-se um politopo contendo 8 vértices.

\subsection{Projeto do controlador robusto $K$ via taxa de decaimento}

Inicialmente é necessário definir o valor da taxa de decaimento para o projeto, após diferentes testes, usa-se $\gamma=$ 1,66. Utilizou-se a biblioteca YALMIP (Lofberg, 2004) e o solver SeDuMi (Sturm, 1999), por meio do software Matlab ${ }^{\circledR}$, para a resolução das desigualdades matriciais do Lema 1.

A matriz $K$ projetada, que contém os ganhos do controlador PID robusto, dada por $K=G P^{-1}$, foi:

$$
K=\left[\begin{array}{lll}
K_{p} & K_{d} & K_{i}
\end{array}\right]=\left[\begin{array}{lll}
0,0691 & 0,0042 & 0,2481
\end{array}\right] .
$$

Na Figura 6, é possível visualizar a localização dos polos do politopo, alocados a esquerda de $\gamma$.

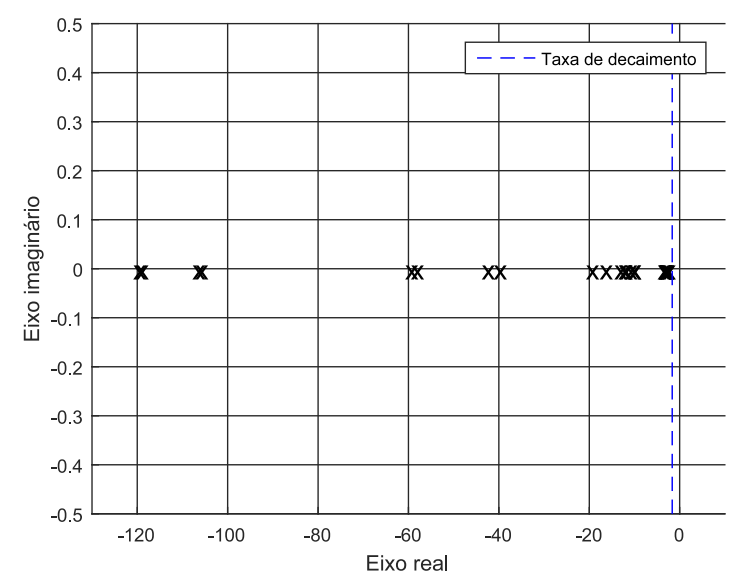

Figura 6. Localização dos polos de malha fechada realimentado por $K$

\section{RESULTADOS DA IMPLEMENTAÇÃO}

Através da Figura 7, observa-se o comportamento da velocidade do motor CC, i.e., a saída do sistema, $y(t)$, diante do sinal de referência, $r(t)$. A ação de controle robusta foi capaz de controlar a velocidade e eliminar erros de regime permanente, mesmo diante de modificações abruptas no sinal de referência aplicado, o qual variou de zero à $300 \mathrm{rpm}$, mediante rampas de aceleração e degraus.

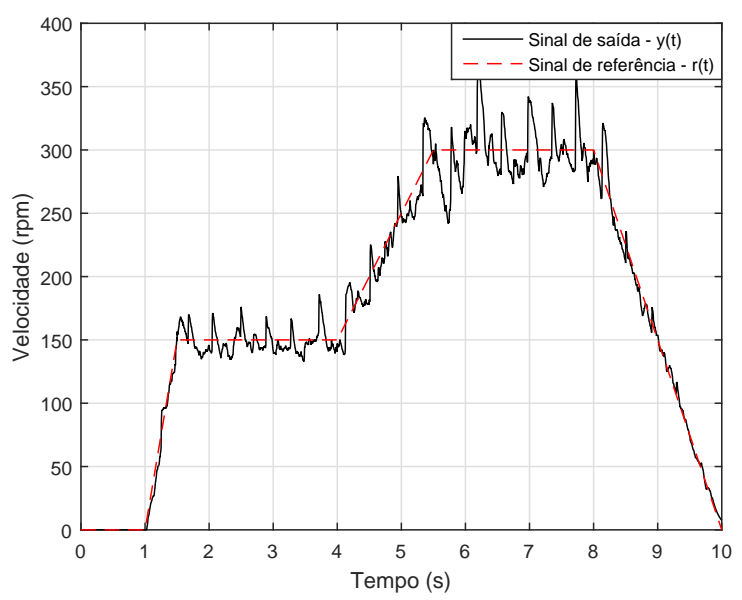

Figura 7. Resposta ao sinal de referência

A Figura 8 mostra o esforço do sinal de controle $u(t)$ para manter a rotação do motor na referência desejada.

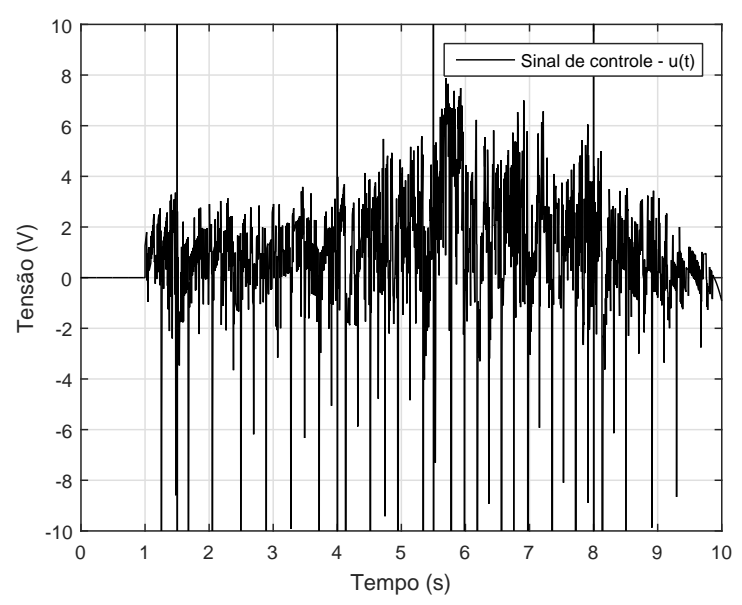

Figura 8. Sinal de controle

Com o objetivo de validar a robustez do controlador, simulou-se uma falha na realimentação $u(t)$ do sistema. A implementação foi realizada atenuando o sinal de realimentação por meio da estrutura de controle desenvolvida no software Simulink ${ }^{\circledR}$. Com isto, o sinal de controle aplicado ao driver de alimentação foi atenuado em $30 \%$ no instante $t=4 \mathrm{~s}$. Neste instante, esperava-se uma queda momentânea na velocidade do motor, e em seguida sua recuperação. Na Figura 9, é possível observar a resposta do sistema diante desta atenuação, verificando-se em $t=4 \mathrm{~s}$ uma leve queda na velocidade.

Devido a ação do controle PID robusto, projetado considerando a possibilidade de falha, o sistema se recupera rapidamente. $\mathrm{O}$ sinal de controle resultante pode ser visualizado na Figura 10, onde verifica-se, em $t=4 \mathrm{~s}$, um elevado pico no sinal de controle, representando a atuação do controlador. 


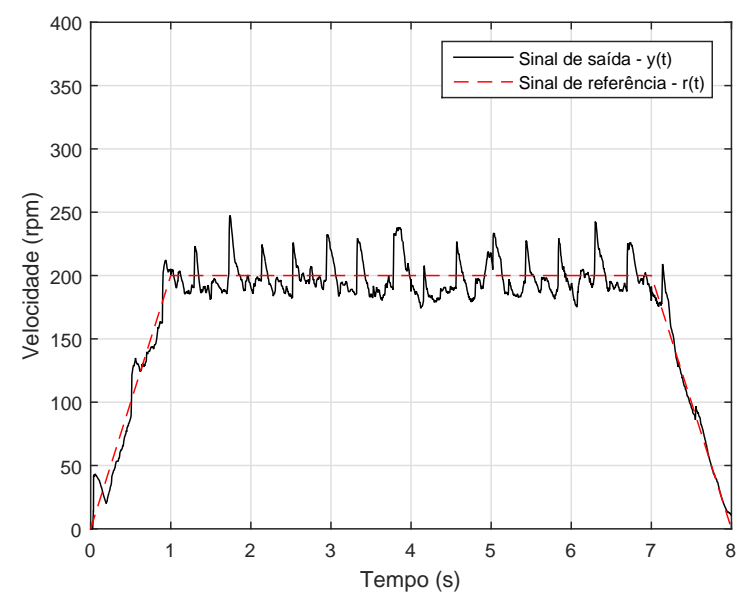

Figura 9. Resposta à falha

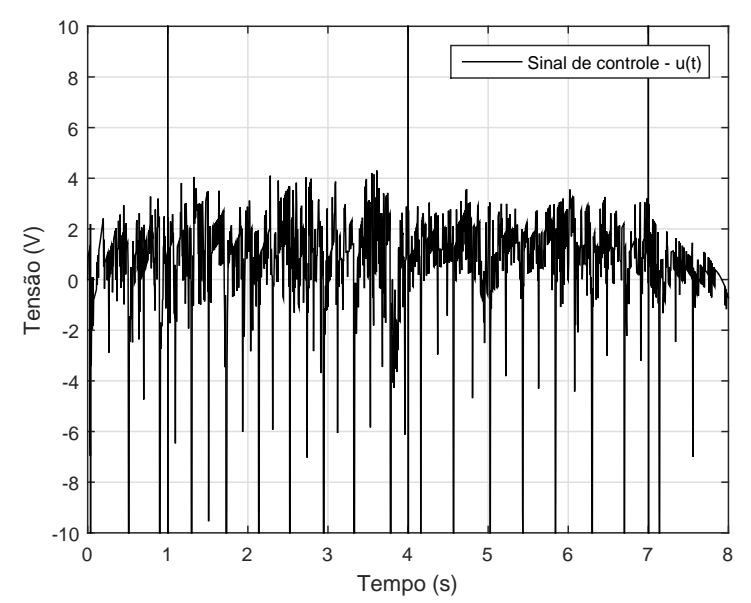

Figura 10. Sinal de controle - Falha

É possível observar nos testes realizados a boa atuação do controlador PID robusto, que garantiu a estabilidade e o bom desempenho do sistema, mesmo diante de uma falha, condição crítica do motor. Uma observação importante é a relação de desempenho com a taxa de decaimento. Compreende-se que, quanto maior seja a taxa de decaimento, mais rápida será a resposta do sistema (tempo de assentamento, tempo de subida), nos fornecendo, portanto, uma abordagem qualitativa de desempenho. Podese acelerar a resposta do sistema, aumentando sua taxa de decaimento, alocando os polos em uma região mais distante do eixo imaginário. Contudo, deve-se atentar que este deslocamento resulta em um maior esforço de controle, exigindo capacidade física de resposta dos atuadores.

\section{CONCLUSÕES}

O objetivo principal deste trabalho foi a implementação de um sistema de controle PID robusto sintonizado via desigualdades matriciais lineares, com resultados validados por meio de testes experimentais.

A implementação se mostrou simples dada a modelagem do sistema de controle, que permitiu controlar o motor à partir de um único estado acessível, a velocidade angular, $y(t)$, sendo os demais estados estimados à partir de $y(t)$.
O método de alocação de polos, via taxa de decaimento, introduziu parâmetros de desempenho ao sistema realimentado, permitindo ao projetista adequar o desempenho de acordo com as singularidades do projeto.

O trabalho exposto possui perspectivas para melhorias e desenvolvimento de trabalhos futuros, já que a metodologia é flexível, facilmente adaptável e com grande possibilidade de ajustes de desempenho. A utilização de técnicas de otimização do controlador, tal como as normas $H_{2}$ e $H_{\infty}$, permitem aprofundar e melhorar a técnica implementada. Pode-se citar também o estudo de sistemas de controle via realimentação derivativa e controladores do tipo LQR.

\section{AGRADECIMENTOS}

Os autores agradecem à UTFPR, Câmpus Cornélio Procópio, e à Coordenação de Aperfeiçoamento de Pessoal de Nível Superior (Capes).

\section{REFERÊNCIAS}

Bensafia, Y., Khettab, K., and Ladaci, S. (2015). Dc-motor velocity control using a robust fractionalized adaptive pi controller. In 2015 16th International Conference on Sciences and Techniques of Automatic Control and Computer Engineering (STA), 622-627.

Bonzanini, J.M. (2017). Estudo de técnicas de controle PID aplicado em motor CC. Trabalho de conclusão de curso - especialização, Universidade Tecnológica Federal do Paraná, Cornélio Procópio.

Boyd, S., El Ghaoui, L., Feron, E., and Balakrishnan, V. (1994). Linear Matrix Inequalities in System and Control Theory, volume 15 of Studies in Applied Mathematics. SIAM, Philadelphia, PA.

Chairinnas, Joelianto, E., Gani, G., and Putri, N.K. (2016). Robust control with linear matrix inequality approach for ship steering problem. In 2016 International Conference on Instrumentation, Control and Automation (ICA), 126-131.

Cheng, C.Y. (2006). Autotuning of PID Controllers - A Relay Feedback Approach. Springer-Verlag London, 2 edition.

Ge, M., Chiu, M.S., and Wang, Q.G. (2002). Robust pid controller design via lmi approach. Journal of process control, 12(1), 3-13.

Hypiusová, M. and Rosinová, D. (2018). Discrete-time robust lmi pole placement for magnetic levitation. In 2018 Cybernetics Informatics (K I), 1-6. doi:10.1109/ CYBERI.2018.8337565.

Lofberg, J. (2004). Yalmip : a toolbox for modeling and optimization in matlab. In 2004 IEEE International Conference on Robotics and Automation (IEEE Cat. No.04CH37508), 284-289.

Ogata, K. (2010). Engenharia de controle moderno. Pearson Prentice Hall, São Paulo, SP, 5 edition.

Sturm, J.F. (1999). Using sedumi 1.02, a matlab toolbox for optimization over symmetric cones. Optimization Methods and Software, 11(1-4), 625-653. URL https : // doi.org/10.1080/10556789908805766.

Zhangbao, X., Jianyong, Y., Dawei, M., and Guichao, Y. (2015). Robust control of dc motors based on disturbance estimation. In 2015 34th Chinese Control Conference (CCC), 4251-4255. 